\title{
Publisher's Note: Two-photon vibrational excitation of air by long-wave infrared laser pulses [Phys. Rev. A 94, 023816 (2016)]
}

\author{
J. P. Palastro, J. Peñano, L. A. Johnson, B. Hafizi, J. K. Wahlstrand, and H. M. Milchberg
}

(Received 11 January 2018; published 26 January 2018)

DOI: 10.1103/PhysRevA.97.019904

This paper was published online on 5 August 2016 with an error in the Acknowledgments. The third sentence in the Acknowledgments should read as "J.K.W. and H.M.M. acknowledge the support of the Air Force Office of Scientific Research (Grants No. FA9550-13-10044 and No. FA9550-16-10121), the National Science Foundation (Grant No. PHY1301948), and the Army Research Office (Grant No. W911NF1410372)." The Acknowledgments have been corrected as of 28 December 2017. The Acknowledgments are incorrect in the printed version of the journal. 